\title{
Difensil Immuno Reduces Recurrence and Severity of Tonsillitis in Children: A Randomized Controlled Trial
}

\author{
Arianna Di Stadio ${ }^{1, *}$, Antonio della Volpe ${ }^{2}$, Fiammetta M. Korsch ${ }^{2}$, Antonietta De Lucia ${ }^{2}$, \\ Massimo Ralli ${ }^{3}$ (D), Francesco Martines ${ }^{4}$ and Giampietro Ricci ${ }^{1}$ \\ 1 Department of Otolaryngology, University of Perugia, 06129 Perugia, Italy; ricci1501@hotmail.com \\ 2 Otology and Cochlear Implant Unit, Santobono-Pausilipon Children's Hospital, 80129 Naples, Italy; \\ antonidellavolpe@yahoo.it (A.d.V.); fiammettakorsch@gmail.com (F.M.K.); toniadelucia@live.it (A.D.L.) \\ 3 Sense Organs Department, Sapienza University of Rome, 00161 Rome, Italy; massimo.ralli@uniroma1.it \\ 4 Biomedicine, Neuroscience and Advanced Diagnostics Department, University of Palermo, \\ 90127 Palermo, Italy; francesco.martines@unipa.it \\ * Correspondence: Arianna.distadio@unipg.it or ariannadistadio@hotmail.com
}

Received: 13 May 2020; Accepted: 26 May 2020; Published: 2 June 2020

\begin{abstract}
Oral supplements (OS) support the immune system in fighting upper airways infection. This study aimed to analyze the effect of Difensil Immuno (DI) on the recurrence of tonsillitis and fever in children. A multicentric randomized clinical trial was conducted. One-hundred and twenty children with chronic tonsillitis were randomly assigned to group A, B or control. Patients in group A were treated with $10 \mathrm{~mL}$ of DI for 90 consecutive days, patients in group B underwent treatment with $15 \mathrm{~mL}$ of DI for 45 consecutive days. The following data were collected at baseline (T0), T1 and T2: tonsillitis and fever episodes, tonsillar volume, blood test results. One-way ANOVA was used to analyze within and between variances. Patients in group A and B statistically improved their clinical parameters (episode of tonsillitis and fever, tonsillar volume) when compared to control group both at T1 and T2. However, T1 variances were more consistent in group A than in group B. All patients in the study groups improved their clinical outcomes. No statistically significant variances were observed in blood parameters both at T1 and T2. Our results suggest that children treated with DI had fewer episodes of tonsillitis and fever and a reduction in their tonsillar volume.
\end{abstract}

Keywords: tonsillitis; treatment; oral supplement; immune system; immune stimulation

\section{Introduction}

The benefic effect of oral supplements (OS) on the immune system has been shown in patients suffering from immunodeficiency [1] and in subjects with cancer [2,3]; some specific vitamins, such as vitamin $\mathrm{D}$, were shown to be able to immunomodulate the immune system of patients with Multiple Sclerosis by reducing neurodegeneration [4].

Vitamins and antioxidant molecules act on the immune system in different ways; for example, some of them increase the number of circulating white cells [5], while others empower the reactivity of the immune response by reducing the level of reactive oxygen species (ROS) known to be dangerous for the immune system [6].

Most studies with OS are conducted in the adult population and studied these compounds in non-infectious diseases [1-3]. On the contrary, a few studies have been performed on children suffering from infectious disease to test the efficacy of OS [7].

Recently, a randomized clinical trial from our group showed that an OS containing Sambucus nigra, zinc, tyndallized Lactobacillus acidophilus (HA122), arabinogalactans, vitamin D, vitamin E 
and vitamin C (commercial name Difensil Immuno (DI), available in Italy and Europe) could improve the outcome of children with otitis media [8]. In this study, DI improved the immune response and reduced the virulence of infection [8].

Each component of DI interacts differently with the immune system. Sambucus nigra inhibits viral replication and increases the production of inflammatory cytokines; vitamin $C$ reduces the circulating oxidative species, whose presence negatively impact on the immune response; vitamin $\mathrm{D}$ promotes differentiation of monocytes/macrophages in their active form and increases the chemotactic and phagocytic capacity of these cells; zinc improves the macrophage phagocytosis ability, the capacity of antigen presentation and signal transmission between these cells and the other belonging to the immune-system; selenium is a potent immune-stimulant able to improve $\mathrm{T}$ cell proliferation, natural killer cell activity and several innate immune cell functions. Finally, Lactobacillus acidophilus and arabinogalactans stimulate the macrophage activity and the production of cytokines.

Children commonly suffer from tonsillitis due to their immature immune system; generally, antibiotics and corticosteroids are used for the treatment of recurrent episodes [9]. The aim of this randomized clinical trial was to analyze the effect of DI on the recurrence of tonsillitis and fever in children.

\section{Materials and Methods}

This multicentric study was conducted in the Otolaryngology departments of the following institutions: Santobono-Pausilipon hospital, University of Perugia and University of Palermo from October 2019 to March 2020. All procedures were approved by the local Institutional Review Board committee of each hospital and were conducted in accordance with the ethical principles outlined in the Declaration of Helsinki. The parents of the participating children signed a written informed consent document authorizing the enrollment of their child in the study.

The three centers used the same criteria and the procedures were standardized before starting the study.

The inclusion criteria were age $<8$, at least 3 episodes of tonsillitis per year with fever, Mackenzie score $>2$, no previous history of adenoidectomy, no current treatment with antibiotics, no history of allergies, no diabetes or severe neurological disease.

In each hospital, patients were randomly assigned by a computer to one of three groups: Group A (GA), Group B (GB) and control Group (CG). GA and GB were treated with OS with immune-stimulating molecules (Sambucus nigra, zinc, tyndallized Lactobacillus acidophilus (HA122), arabinogalactans, vitamin D, vitamin E and vitamin C) (Difensil Immuno, Humana Italia S.p.A.).

GA included patients who were treated with $10 \mathrm{~mL}$ of DI for 90 consecutive days; GB patients underwent treatment with $15 \mathrm{~mL}$ of DI for 45 consecutive days; and lastly CG did not receive any therapy. DI was always administered in a single dose in the morning during breakfast.

A dose of $10 \mathrm{~mL}$ of DI contains $112 \mathrm{mg}$ of Sambucus nigra, $7.5 \mathrm{mg}$ of zinc, $1 \times 10^{7}$ tyndallized Lactobacillus acidophilus (HA122), $10 \mathrm{mg}$ of arabinogalactans, $10 \mathrm{mcg}$ of vitamin D, $30 \mathrm{mg}$ of vitamin $\mathrm{E}$ and $90 \mathrm{mg}$ of vitamin C. A dose of $15 \mathrm{~mL}$ of DI contains $183 \mathrm{mg}$ of Sambucus nigra, $11.2 \mathrm{mg}$ of zinc, $1.5 \times 10^{7}$ tyndallized Lactobacillus acidophilus (HA122), $15 \mathrm{mg}$ of arabinogalactans, $15 \mathrm{mcg}$ of vitamin $\mathrm{D}, 45 \mathrm{mg}$ of vitamin $\mathrm{E}$ and $135 \mathrm{mg}$ of vitamin $\mathrm{C}$.

In cases where fever exceeded $38{ }^{\circ} \mathrm{C}$, children were treated with paracetamol $(500 \mathrm{mg})$ and antibiotics if fever persisted over three days.

Patients in the CG followed a "wait and see approach"; children were treated with anti-inflammatory drugs in cases of a fever episode over $38^{\circ} \mathrm{C}$, and antibiotics if the condition did not resolve within three days. Corticosteroids were never used.

Outcome measures included: number of episodes of tonsillitis during the observation period and episodes of fever during the period of follow-up, inspection of tonsil aspect and classification following the Mackenzie classification (Figure 1), red cell count, white cell count, hemoglobin ( $\mathrm{Hb})$, 
polymerase chain reaction (PCR) value, erythrocyte sedimentation rate (ESR), antistreptolysin O titer (ASLOT), pro-calcitonin, rheumatologic test (RT).

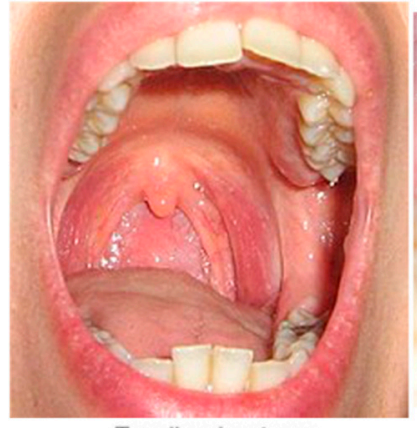

Tonsils, size 1, are hidden within the pillars.

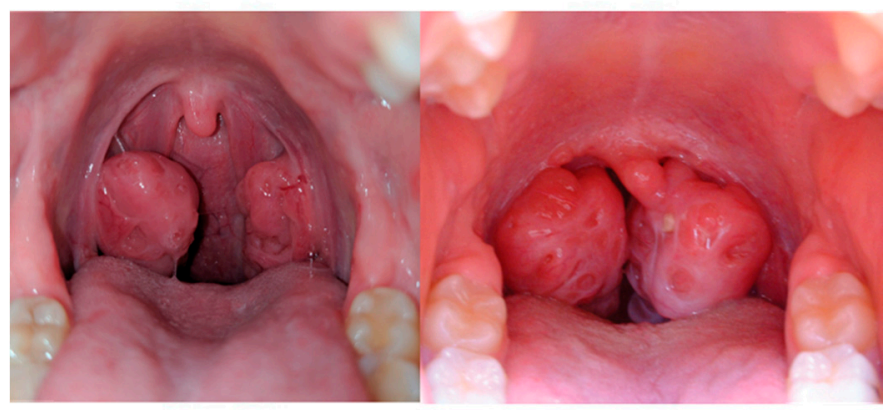

Tonsils, size 3 , extend beyond the pillars, but not to the midline.

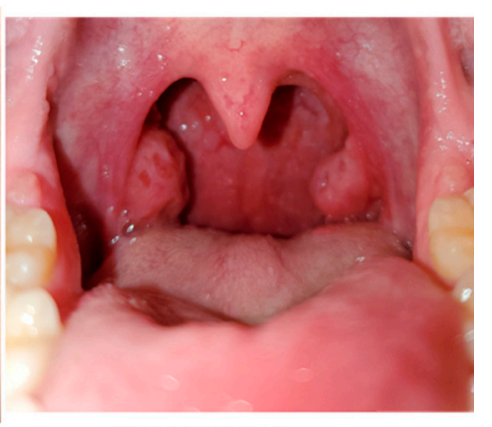

Tonsils, size 2 extend to the pillars.

Tonsils, size 4 , extend to the midline.

Figure 1. The image shows details of the Mackenzie classification. We used pictures from our sample to detail the differences between different severities of tonsillar aspect.

Three time points were identified: $\mathrm{T} 0=$ before treatment (baseline), $\mathrm{T} 1=45$ days after treatment and $\mathrm{T} 2=90$ days after treatment; at each follow-up all outcome measures were collected.

Episodes of tonsillitis and fever at T0 were counted by considering the last 3 months (90 days) before the beginning of the study.

One-hundred twenty subjects were enrolled; each center recruited 40 patients. In detail, Group A included 40 children, average age $=6$ years (SD: 2.6; CI 95\%: 3-12); 25 were females and 15 males. Group B included 41 children, average age $=6$ years (SD: 2.5; CI: 3-12), 22 were females and 19 males. Control group included 39 children, average age $=5.6$ years (SD: 2.3; CI 95\%: 3-12), 20 females and 19 males. None of the patients underwent antibiotic therapy, 15 days being included in the study for all the observation period up to one week after the end of the study.

All patients completed the study and none of them dropped or missed follow-up visits.

\section{Statistical Analysis}

The statistical analysis was performed using STATA ${ }^{\circledR}$. One-way ANOVA was used to evaluate the scores variation within each group (GA, GB and CG) at three time points (T0, T1 and T2) for the number of episodes of tonsillitis and fever. The same test was repeated to evaluate the variance of the tonsils (Mackenzie) and the blood parameters. A Bonferroni-Holms ad hoc test was performed for each one-way ANOVA. A $p$ value $<0.05$ was considered statistically significant. 


\section{Results}

3.1. Treatment Results "within" Group Comparison

\subsubsection{Group A}

None of the patients needed antibiotics treatment (Figure 2 and Table 1).

\section{Within-group results}

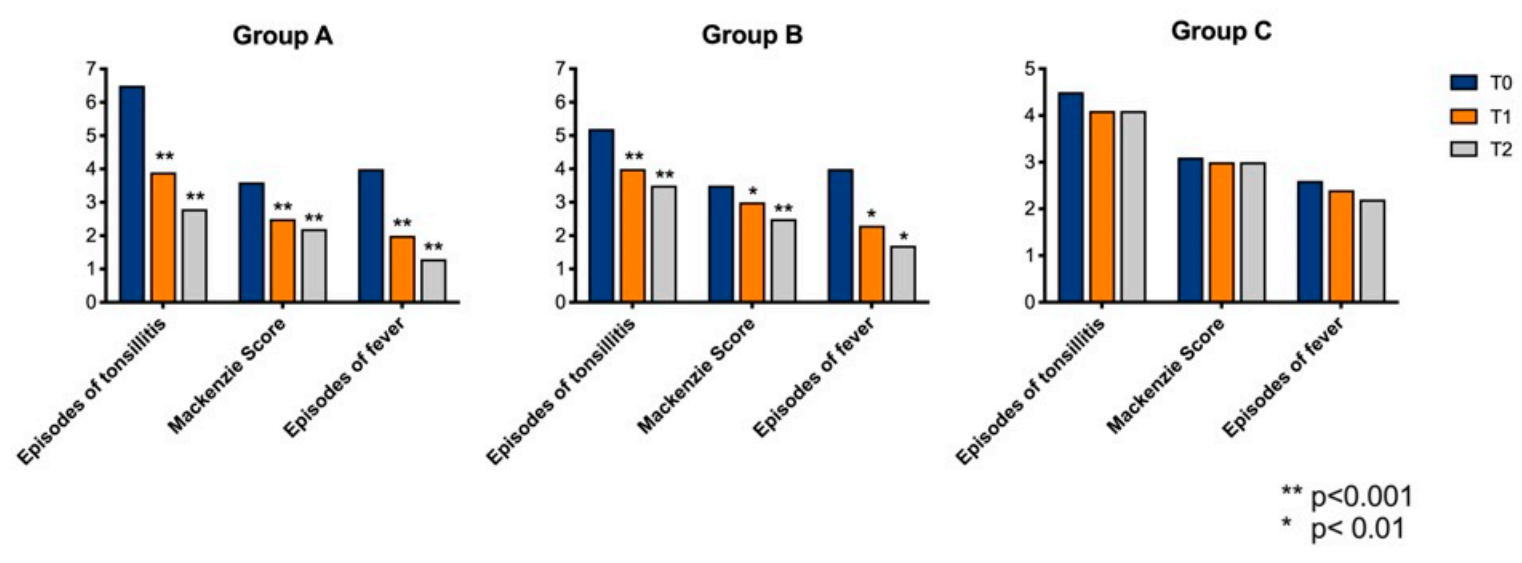

Figure 2. Variation within each group by comparing T0, T1 and T2 for episodes of tonsillitis, Mackenzie Score and episodes of fever.

Table 1. Average of clinical data in Group A observed at the three different follow-up visits.

\begin{tabular}{lccc}
\hline Group A & T0 & T1 & T2 \\
\hline \multirow{2}{*}{ Episodes of tonsillitis } & 6.5 (SD: $1 ;$ & 3.9 (SD: $1 ;$ & 2.8 (SD: $0.9 ;$ \\
\multirow{2}{*}{ Mackenzie Score } & CI 95\%:4-8) & CI 95\%:2-5) & CI 95\%:1-4) \\
\multirow{2}{*}{ Episodes of fever } & 3.6 (SD: 0.5; & 2.5 (SD: $1 ;$ & 2.2 (SD: 0.7; \\
& CI 95\%:3-4) & CI 95\%:1-4) & CI 95\%: $1-3)$ \\
& 4 (SD: 1 & 2 (SD: $0.7 ;$ & (SD: $0.6 ;$ \\
\hline
\end{tabular}

Statistically significant variations were observed by comparing T0, T1 and T2 follow-up for the following parameters:

(1) Number of tonsillitis episode (ANOVA: $p<0.0001)$ between T0 and T1 (BH: $p<0.0001$ ), between T0 and T2 (BH: $p<0.0001)$ and T1 and T2 (BH: $p<0.0001)$.

(2) Mackenzie score (ANOVA: $p<0.0001$ ). In particular a significant variance was observed between T0 and T1 (HB: $p<0.0001$ ), T0 and T2 (HB: $p<0.0001)$. No statistically significant variances were observed between T1 and T2 (HB: $p=0.3$ ).

(3) Number of fever episode (ANOVA: $p<0.0001$ ). The variance was statistically significant by comparing T0 and T1 (HB: $p<0.0001)$, T0 and T2 (HB: $p<0.0001)$ and T1 and T2 (HB: $p<0.0001)$.

No statistically significant variations were observed by comparing children at T0, T1 and T2 for the following parameters: red cell count (ANOVA: $p=1$ ), white cell count (ANOVA: $p=1)$, Hb (ANOVA: $p=1$ ), PCR value (ANOVA: $p=0.6$ ), ESR (ANOVA: $p=0.4)$, ASLOT (ANOVA: $p=1$ ), pro-calcitonin (ANOVA: $p=0.9$ ), RT (ANOVA: $p=1$ ).

\subsubsection{Group B}

None of the patients were treated with antibiotics (Figure 2 and Table 2). 
Table 2. Average of clinical data in Group B observed at the three different follow-up visits.

\begin{tabular}{lccr}
\hline Group B & T0 & T1 & T2 \\
\hline \multirow{2}{*}{ Episodes of tonsillitis } & 5,2 (SD: 1.2 & 4 (SD: $1.1 ;$ & 3.3 (SD: 0.9; \\
\multirow{2}{*}{ Mackenzie Score } & CI 95\%: 4-8) & CI 95\%: 2-7) & CI 95\%: 2-5) \\
\multirow{2}{*}{ Episodes of fever } & 3.5 (SD: 0,6 & 3 (SD: 0.6; & 2.5 (SD: 0.5; \\
& CI 95\%:2-4) & CI 95\%:2-4) & CI 95\%:2-4) \\
& 4 (SD 0.1 & 2,3 (SD: $0.7 ;$ & 1.7 (SD: $0.8 ;$ \\
\hline
\end{tabular}

Statistically significant variations were observed by comparing T0, T1 and T2 follow-up for the following parameters:

(1) Number of tonsillitis episode (ANOVA: $p<0.0001$ ) between T0 and T1 (BH: $p<0.0001$ ), between $\mathrm{T} 0$ and T2 (BH: $p<0.0001)$. No statistically significant variances were observed between $\mathrm{T} 1$ and T2 (HB: $p=0.07$ ).

(2) Mackenzie score (ANOVA: $p<0.0001$ ). In particular, a significant variance was observed between T0 and T2 (HB: $p<0.0001$ ) and between T1 and T2 (HB: $p=0.01$ ). No statistically significant variances were observed between $\mathrm{T} 0$ and $\mathrm{T} 1$ (HB: $p=0.06$ ).

(3) Number of fever episode (ANOVA: $p=0.002$ ). The variance was statistically significant by comparing T0 and T1 (HB: $p<0.01$ ), T0 and T2 (HB: $p<0.01$ ) and T1 and T2 (HB: $p<0.01$ ).

No statistically significant variations were observed by comparing children at T0, T1 and $\mathrm{T} 2$ for the following parameters: red cell count (ANOVA: $p=1$ ), white cell count (ANOVA: $p=1$ ), Hb (ANOVA: $p=1$ ), PCR value (ANOVA: $p=0.9$ ), ESR (ANOVA: $p=0.5$ ), ASLOT (ANOVA: $p=1$ ), pro-calcitonin (ANOVA: $p=0.9$ ), RT (ANOVA: $p=1$ ).

\subsubsection{Control Group}

Fifty per-cent of children needed antibiotics treatment (Figure 2 and Table 3).

Table 3. Average of clinical data in group B observed at the three different follow-up visits.

\begin{tabular}{lccc}
\hline Control Group & T0 & T1 & T2 \\
\hline \multirow{2}{*}{ Episodes of tonsillitis } & 4.5 (SD: 0.9 & 4.1 (SD: 0.9 & 4.1 (SD: $1 ;$ \\
& CI 95\%: 3-6) & CI 95\%:2-5) & CI 95\%:2-6) \\
Mackenzie Score & 3 (SD: 0.5 & 3 (SD: 0.6 & 3 (SD: 0.6 \\
\multirow{2}{*}{ Episodes of fever } & CI 95\%:2-4) & CI 95\%:2-4) & CI 95\%:2-4) \\
& 2.6 (SD: 0.8 & 2.4 (SD: 0.6 & 2.2 (SD: 0.8 \\
& CI 95\%: $4-8)$ & CI 95\%:2-4) & CI 95\%:1-4) \\
\hline
\end{tabular}

No statistically significant variations were observed by comparing children at $\mathrm{T} 0$, $\mathrm{T} 1$ and $\mathrm{T} 2$ for the following parameters: number tonsillitis episodes between the three follow-up (ANOVA: $p=0.2$ ), Mackenzie scores (ANOVA: $p=0.9$ ), fever episodes (ANOVA: $p=0.2$ ) red cell count (ANOVA: $p=1$ ), white cell count (ANOVA: $p=1$ ), Hb (ANOVA: $p=1$ ), PCR value (ANOVA: $p=0.6$ ), ESR (ANOVA: $p=0.9$ ), ASLOT (ANOVA: $p=1$ ), pro-calcitonin (ANOVA: $p=0.7$ ), RT (ANOVA: $p=1$ ).

\subsection{Treatment Results "between" Group Comparison}

Statistically significant variances were observed between GA, GB and CG for the following parameters:

\subsubsection{Comparison at $\mathrm{T} 1$}

(1) Number of tonsillitis episode (ANOVA: $p<0.0001$ ). We observed statistically significant variances between GA (average: 3.9; SD: 1; CI 95\%: 2-5) and CG (average: 4.1; SD: 0.9; CI 95\%: 2-5) 
(BH: $p<0.0001$ ), but not between GB (average: 4; SD: 1.1; CI 95\%: 2-7) and CG (BH: $p>0.05$ ). Statistically significant variances were observed between GA and GB (HB: $p<0.0001)$.

(2) Mackenzie score (ANOVA: $p<0.0001$ ). GA (average: 2.5; SD: 1; CI 95\%: 1-4) showed statistically significant variances with CG (average: 3; SD: 0.6; CI 95\%: 2-4) (HB: $p=0.0007$ ), while no statistically significant variances were observed between GB (average: 3; SD: 0.6; CI 95\%: 2-4) and CG (HB: $p>0.05)$. Statistically significant variance was observed by comparing GA and GB (HB: $p<0.00001)$.

(3) Number of fever episode (ANOVA: $p=0.002$ ). The variance was statistically significant by comparing GA (average: 2; SD: 0.7; CI 95\%: 1-3) with CG (average: 2.4; SD: 0.7; CI 95\%: 1-4) (HB: $p=0.001$ ) and GB (average: 2.3; SD: 0.7; CI 95\%: 1-4) with CG (HB: $p=0.002$ ). No statistically significant differences were observed between GA and GB.

No statistically significant variations were observed by comparing GA, GB and CG at T1 for the following parameters: red cell count (ANOVA: $p=1$ ), white cell count (ANOVA: $p=1$ ), Hb (ANOVA: $p=1$ ), PCR value (ANOVA: $p=0.7$ ), ESR (ANOVA: $p=0.7$ ), ASLOT (ANOVA: $p=0.9$ ), pro-calcitonin (ANOVA: $p=0.9$ ), RT (ANOVA: $p=1$ ) (Figure 3).

\section{Between-group results}

T1

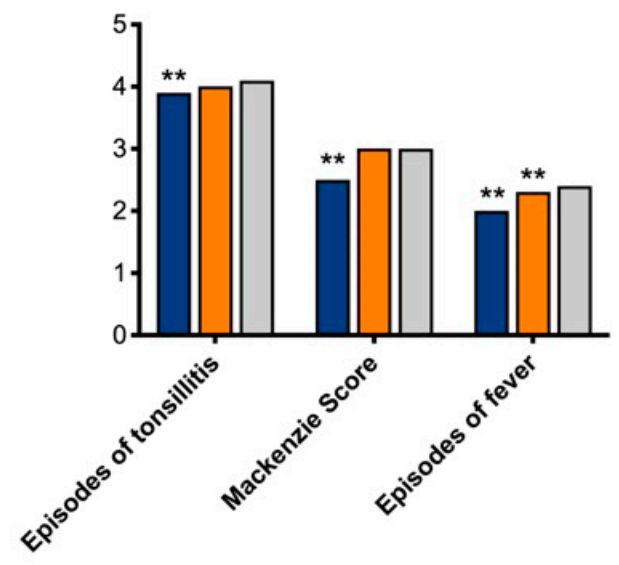

T2

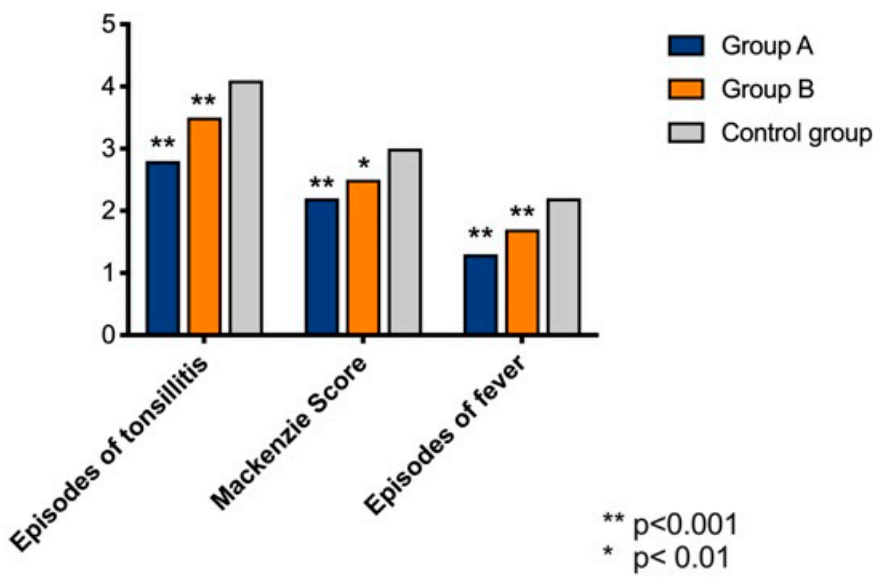

Figure 3. Differences of the analyzed variables between Group A, Group B and Control Group. Patients in Group A obtained a statistically significant improvement from T0 as detailed in the results.

\subsubsection{Comparison at $\mathrm{T} 2$}

(1) Number of tonsillitis episode (ANOVA: $p<0.0001$ ). By comparing GA (average: 2.8; SD: 0.8; CI 95\%: 1-4) and CG (average: 4.1; SD: 1; CI 95\%: 2-6) we observed statistically significant variances at T2 (HB: $p<0.0001$ ) and the same results were observed when comparing GB (average: 3.3; SD: 0.9; CI 95\%: 2-5) with CG (HB: $p=0.0002)$. No statistically significant variances were observed between GA and GB.

(2) Mackenzie score (ANOVA: $p<0.0001$ ). GA (average: 2.3; SD: 0.7; CI 95\%: 1-3) showed statistically significant variances with CG (average: 3; SD: 0.6; CI 95\%: 2-4) at T2 (HB: $p=0.0001$ ), as well as GB (average: 2.5 ; SD: 0.5 ; CI 95\%: $2-3$ ) versus CG (HB: $p=0.01$ ). A statistically significant variance was observed by comparing GA and GB (HB: $p=0.003)$.

(3) Number of fever episode (ANOVA: $p=0.002$ ). The variance was statistically significant by comparing GA (average: 1.3; SD: 0.6; CI 95\%: 0-2) and CG (average: 2.2; SD: 0.9; CI 95\%: 1-3) at T2 (HB: $p=0.001$ ) and GB (average: 1.7 ; SD: 0.7 ; CI 95\%: $1-3$ ) and CG at T2 (HB: $p=0.002$ ). No statistically significant differences were observed between GA and GB. 
No statistically significant variations were observed by comparing GA, GB and CG at T2 for the following parameters: red cell count (ANOVA: $p=1$ ), white cell count (ANOVA: $p=1$ ), Hb (ANOVA: $p=1$ ), PCR value (ANOVA: $p=0.7$ ), ESR (ANOVA: $p=0.7$ ), ASLOT (ANOVA: $p=0.9$ ), pro-calcitonin (ANOVA: $p=0.9$ ), RT (ANOVA: $p=1$ ) (Figure 3 ).

\section{Discussion}

Our results show that DI reduced the recurrence of tonsillitis and the fever episodes over a period of three months and reduced the average volume of tonsils. Furthermore, children who used DI never needed antibiotics treatment. Despite these clinical results, the use of OS did not modify the blood parameters of inflammation (PCR, ESR and pro-calcitonin). However, the supplement did not affect the other blood parameters.

All patients ("between analysis"), independently from the therapeutic scheme used, showed a better outcome than CG, supporting the use of DI as a stimulator of natural immune answers in children.

Patients treated with $10 \mathrm{~mL}$ of OS for 90 consecutive days (GA) showed an improvement after 45 days, while patients who underwent $15 \mathrm{~mL} \times 45$ days (GB) significatively improved their outcome after 90 days. This result showed that the use of a low dosage for a longer period could reduce the episode and the severity of tonsillitis better than a higher dosage for a short time, as has already been shown for other types of OS [10].

On the other hand, patients in GB were able to improve their condition after the end of the treatment; it could be speculated that a high dosage, despite less efficiency in the short time, could be released slowly [11] and improve patients' outcomes after treatment suspension.

The "within" analysis group partially confirmed the observations of the "between groups" analysis; in fact, both therapeutic schemes improved patients' outcomes.

Patients in GA showed improvements in all outcomes (episode of tonsillitis, Mackenzie score and fever episode) after 45 days of treatment and this improvement was consistent at the second follow-up (90 days); children in GB presented a reduced number of tonsillitis cases and fever episodes at T1 without a statistically significant change in the tonsil volume; this outcome improved at T2. In addition, patients in GB showed a further reduction of tonsillitis and fever episodes after 90 days, by reaching results similar to those observed in GA.

We speculate that the delay in tonsil volume reduction in GB could be related to the high concentration of DI, which stimulates an increase in the local immune response [12] with consequent persistence of tonsillar hypertrophy. This initial over-stimulation of white cells in the Waldeyer ring could explain the improvement observed in these patients after the end of oral supplementation. We previously showed the efficacy of this specific OS to improve the immune answer in the Waldeyer ring and to determine an important improvement of otitis media in children [8].

Overall, our results suggest that a long-term treatment with $10 \mathrm{~mL}$ of OS is better than a shorter one, although the short time, high-dosage therapeutic scheme maintains a very good efficacy after treatment suspension.

We speculate that DI could have stimulated the immune system function [13-16] thanks to the synergic effect of its different components. S. nigra, which has been confirmed as an efficient molecule for inhibiting viral replication in the early stages of infection [17] was fundamental to reduce the episode of tonsillitis and consequently to decrease fever recurrence. A, C, D and E vitamins contributed to the action of the $S$. nigra by increasing mucosal IgA (A and E) $[18,19]$ and by actively stimulating macrophage and lymphocyte activity $[5,20]$, thus making children more resistant to viral infection in the upper respiratory tract (URT). The reduction of the number of infections progressively allowed the reduction of tonsil volume.

Lactobacillus acidophilus, another component of the OS, can improve the immune response thanks to the reduction of concentration of ROS, known as a depressor of the immune system [6]. In addition, lactobacillus inhibits the penetrance of virus inside the cells by further contributing to 
protect URT from common viral infection [21] and has the capacity to inhibit the adhesion and the growth of gram-negative bacteria [22] by protecting the subject from viral and bacterial aggression.

The results of immune-stimulating OS treatment in children reported in this study are consistent with the results observed in adults [23-25] and with a previous study conducted in children in which the authors showed the efficacy of this OS due to its ability to reduce the volume of the adenoid tissue indicative of the resolution of the infective/inflammatory process [26].

However, because of the short duration of this study, additional studies with a longer follow-up are necessary to confirm these preliminary results. In fact, longer studies could evaluate the effect of DI on immune-system cells and could evaluate whether DI could be tolerated for longer periods without interruption.

\section{Conclusions}

Our study results suggest that OS can be a valid tool to reduce the recurrence of tonsillitis and fever episodes in children. In our patients, this natural compound showed a positive effect on the chronic disease without side effects. Although this is the first clinical trial conducted on children with recurrent tonsillitis to evaluate the efficacy of DI, we already successfully tested this compound on children with otitis media.

Author Contributions: Conceptualization, A.D.S. and A.d.V.; Methodology, A.D.S., A.d.V. and F.M.K.; Software, A.D.S.; Validation, A.D.S., A.d.V. and G.R.; Formal Analysis, A.D.S. and M.R.; Investigation, A.d.V., A.D.L., F.M. and G.R.; Data Curation, A.D.S., A.d.V. and M.R.; Writing-Original Draft Preparation, A.D.S., A.d.V. and M.R.; Writing-Review and Editing, G.R.; Supervision, G.R., A.D.S. and A.d.V.; Funding Acquisition, A.d.V. and G.R. All authors have read and agreed to the published version of the manuscript.

Funding: This research received no external funding. Number. PGNAPA 1357911.

Conflicts of Interest: The authors declare no conflict of interest.

\section{References}

1. Ceccarelli, G.; Statzu, M.; Santinelli, L.; Pinacchio, C.; Bitossi, C.; Cavallari, E.N.; Vullo, V.; Scagnolari, C.; $\mathrm{d}^{\prime}$ Ettorre, G. Challenges in the management of HIV infection: Update on the role of probiotic supplementation as a possible complementary therapeutic strategy for cART treated people living with HIV/AIDS. Expert Opin. Biol. Ther. 2019, 19, 949-965. [CrossRef] [PubMed]

2. Ledda, A.; Belcaro, G.; Dugall, M.; Luzzi, R.; Hosoi, M.; Feragalli, B.; Cotellese, R.; Cosentino, V.; Cosentino, M.; Eggenhoffner, R.; et al. A natural pharma standard supplement formulation to control treatment-related toxicity and oxidative stress in genitourinary cancer: A preliminary study. Eur. Rev. Med. Pharm. Sci. 2017, $21,4196-4202$.

3. Harvie, M. Nutritional supplements and cancer: Potential benefits and proven harms. Am. Soc. Clin. Oncol. Educ. Book 2014, 34, e478-e486. [CrossRef]

4. McLaughlin, L.; Clarke, L.; Khalilidehkordi, E.; Butzkueven, H.; Taylor, B.; Broadley, S.A. Vitamin D for the treatment of multiple sclerosis: A meta-analysis. J. Neurol. 2018, 265, 2893-2905. [CrossRef]

5. Konijeti, G.G.; Arora, P.; Boylan, M.R.; Song, Y.; Huang, S.; Harrell, F.; Newton-Cheh, C.; O’Neill, D.; Korzenik, J.; Wang, T.J.; et al. Vitamin D Supplementation Modulates T Cell-Mediated Immunity in Humans: Results from a Randomized Control Trial. J. Clin. Endocrinol. Metab. 2016, 101, 533-538. [CrossRef] [PubMed]

6. Li, C.Y.; Lin, H.C.; Hsueh, K.C.; Wu, S.F.; Fang, S.H. Oral administration of Lactobacillus salivarius inhibits the allergic airway response in mice. Can. J. Microbiol. 2010, 56, 373-379. [CrossRef] [PubMed] 
7. Popovych, V.; Koshel, I.; Malofiichuk, A.; Pyletska, L.; Semeniuk, A.; Filippova, O.; Orlovska, R. A randomized, open-label, multicenter, comparative study of therapeutic efficacy, safety and tolerability of BNO 1030 extract, containing marshmallow root, chamomile flowers, horsetail herb, walnut leaves, yarrow herb, oak bark, dandelion herb in the treatment of acute non-bacterial tonsillitis in children aged 6 to 18 years. Am. J. Otolaryngol. 2019, 40, 265-273. [PubMed]

8. Della Volpe, A.; Ricci, G.; Ralli, M.; Gambacorta, V.; De Lucia, A.; Minni, A.; Pirozzi, C.; Paccone, M.; Pastore, V.; Di Stadio, A. The effects of oral supplements with Sambucus nigra, Zinc, Tyndallized Lactobacillus acidophilus (HA122), Arabinogalactans, vitamin D, vitamin E and vitamin C in otitis media with effusion in children: A randomized controlled trial. Eur. Rev. Med. Pharm. Sci. 2019, 23, 6360-6370.

9. Stelter, K. Tonsillitis and sore throat in children. GMS Curr. Top. Otorhinolaryngol. Head Neck Surg. $2014,13$. [CrossRef]

10. Siani, A.; Strazzullo, P.; Russo, L.; Guglielmi, S.; Iacoviello, L.; Ferrara, L.A.; Mancini, M. Controlled trial of long-term oral potassium supplements in patients with mild hypertension. Br. Med. J. (Clin. Res. Ed.) 1987, 294, 1453-1456. [CrossRef]

11. Minutolo, M.; Chiaiese, P.; Di Matteo, A.; Errico, A.; Corrado, G. Accumulation of Ascorbic Acid in Tomato Cell Culture: Influence of the Genotype, Source Explant and Time of In Vitro Cultivation. Antioxidants 2020, 7, 222. [CrossRef] [PubMed]

12. Elenius, V.; Palomares, O.; Waris, M.; Turunen, R.; Puhakka, T.; Rückert, B.; Vuorinen, T.; Allander, T.; Vahlberg, T.; Akdis, M.; et al. The relationship of serum vitamins A, D, E and LL-37 levels with allergic status, tonsillar virus detection and immune response. PLOS ONE 2017, 12, e0172350. [CrossRef] [PubMed]

13. Shaik-Dasthagirisaheb, Y.B.; Varvara, G.; Murmura, G.; Saggini, A.; Caraffa, A.; Antinolfi, P.; Tete, S.; Tripodi, D.; Conti, F.; Cianchetti, E.; et al. Role of vitamins D, E and C in immunity and inflammation. J. Biol. Regul. Homeost Agents 2013, 27, 291-295. [PubMed]

14. Bergman, P.; Lindh, A.U.; Bjorkhem-Bergman, L.; Lindh, J.D. Vitamin D and Respiratory Tract Infections: A Systematic Review and Meta-Analysis of Randomized Controlled Trials. PLoS ONE 2013, 8, e65835. [CrossRef] [PubMed]

15. Chen, H.; Zhuo, Q.; Yuan, W.; Wang, J.; Wu, T. Vitamin A for preventing acute lower respiratory tract infections in children up to seven years of age. Cochrane Database Syst Rev. 2008. [CrossRef]

16. Vandenplas, Y.; Veereman-Wauters, G.; De Greef, E.; Peeters, S.; Casteels, A.; Mahler, T.; Devreker, T.; Hauser, B. Probiotics and prebiotics in prevention and treatment of diseases in infants and children. J. Pediatr. 2011, 87, 292-300. [CrossRef]

17. Muhamed, P.K.; Vadstrup, S. [Zinc is the most important trace element]. Ugeskr. Laeger 2014, 17, V11120654.

18. Sirisinha, S. The pleiotropic role of vitamin A in regulating mucosal immunity. Asian Pac. J. Allergy Immunol. 2015, 33, 71-89.

19. Amazan, D.; Cordero, G.; Lopez-Bote, C.J.; Lauridsen, C.; Rey, A.I. Effects of oral micellized natural vitamin E (D-alpha-tocopherol) v. synthetic vitamin E (DL-alpha-tocopherol) in feed on alpha-tocopherol levels, stereoisomer distribution, oxidative stress and the immune response in piglets. Animal 2014, 8, 410-419. [CrossRef]

20. Braicu, C.; Mehterov, N.; Vladimirov, B.; Sarafian, V.; Nabavi, S.M.; Atanasov, A.G.; Berindan-Neagoe, I. Nutrigenomics in cancer: Revisiting the effects of natural compounds. Semin. Cancer Biol. 2017, 46, 84-106. [CrossRef]

21. Prado Acosta, M.; Geoghegan, E.M.; Lepenies, B.; Ruzal, S.; Kielian, M.; Martinez, M.G. Surface (S) Layer Proteins of Lactobacillus acidophilus Block Virus Infection via DC-SIGN Interaction. Front. Microbiol. 2019, 10, 810. [CrossRef] [PubMed]

22. Inturri, R.; Stivala, A.; Furneri, P.M.; Blandino, G. Growth and adhesion to HT-29 cells inhibition of Gram-negatives by Bifidobacterium longum BB536 e Lactobacillus rhamnosus HN001 alone and in combination. Eur. Rev. Med. Pharm. Sci. 2016, 20, 4943-4949.

23. Principi, N.; Esposito, S. Severe influenza in children: Incidence and risk factors. Expert Rev. Anti. Infect. Ther. 2016, 14, 961-968. [CrossRef] [PubMed]

24. Frei, R.; Akdis, M.; O'Mahony, L. Prebiotics, probiotics, synbiotics, and the immune system: Experimental data and clinical evidence. Curr. Opin. Gastroenterol. 2015, 31, 153-158. [CrossRef] [PubMed] 
25. D'Angelo, C.; Reale, M.; Costantini, E. Microbiota and Probiotics in Health and HIV Infection. Nutrients 2017, 9, 615. [CrossRef] [PubMed]

26. Loftis, L. Acute infectious upper airway obstructions in children. Semin. Pediatr. Infect. Dis. 2006, 17, 5-10. [CrossRef]

(c)

(C) 2020 by the authors. Licensee MDPI, Basel, Switzerland. This article is an open access article distributed under the terms and conditions of the Creative Commons Attribution (CC BY) license (http://creativecommons.org/licenses/by/4.0/). 